\title{
Research and Application of Unstructured Data Acquisition and Retrieval Technology
}

\author{
Zhenjiang Lei ${ }^{1, *}$, Lin Qiao ${ }^{2}$, Lina $\mathrm{Cao}^{3}$, Liang Ning ${ }^{4}$, Zhuangguan Yang ${ }^{2}$ \\ ${ }^{1}$ ICT Department, State Grid Liaoning Electric Power Supply Co., Ltd: Shenyang 110006, China \\ ${ }^{2}$ Operation \& Maintenance Center, State Grid Liaoning Information and Communication Company: Shenyang \\ 110006 China \\ ${ }^{3}$ Personnel Director Department, State Grid Liaoning Electric Power Supply Co., Ltd: Shenyang 110006, \\ China \\ ${ }^{4}$ Engineering Center, State Grid Liaoning Information and Communication Company: Shenyang 110006, \\ China \\ *Corresponding Author email: leizj@ln.sgcc.com.cn
}

Keywords: Unstructured; Data acquisition; Retrieval technology

Abstract: This paper takes unstructured data acquisition and retrieval technology as the research objects. It firstly expounds the related content of unstructured data acquisition, and then, combining the related system, carried out an in-depth study on the application of unstructured data acquisition and retrieval technology, aiming to offer some significance for the work of related personnel.

\section{Introduction}

In the current information management, structured and unstructured data is the key for information description, in which unstructured data mainly refers to data that is uncertain and not organized according to any data format, such as mails, Word documents, etc. In the daily life, unstructured data is more common, and with the development of modern information technology, the number of unstructured data will increase further, so we must attach due importance to the research on unstructured data acquisition and retrieval technology, so as to better meet the needs of the society.

\section{Collection of unstructured data}

When a worker obtains unstructured data, at this time, the unstructured data is generally independent files, which do not have stipulated structure, so it cannot be standardized. Specific software is needed to be applied to successfully read the problem, which impose am impact on the unstructured data management and analysis ${ }^{[1]}$. So in the current work, in order to be able to obtain the content of unstructured data more conveniently, it is necessary to adopt the corresponding data acquisition and retrieval technology to ensure the data processing requirements.

\section{Application and analysis of data collection and retrieval technology}

In this research, we will combine the related system and carry out in-depth research on the collection and retrieval technology of unstructured data.

\subsection{System requirements and development environment}

In order to better obtain the content of unstructured data, it is necessary to implement different function modules and ensure that all the unstructured data of the system data can be read in a short time. In this case, the operational requirements in the current information transmission environment must be fully taken into account. According to the requirements of general unstructured data acquisition, the requirement structure of the management module function of this system is shown in 
table 1.

Table 1 Management requirements for unstructured functional modules

\begin{tabular}{cccc}
\hline $\begin{array}{c}\text { Functional } \\
\text { classification }\end{array}$ & $\begin{array}{c}\text { Feature } \\
\text { entries }\end{array}$ & $\begin{array}{c}\text { Specific } \\
\text { description }\end{array}$ & Supplementary notes \\
\hline $\begin{array}{c}\text { Information } \\
\text { that needs to } \\
\text { be read }\end{array}$ & $\begin{array}{c}\text { Data } \\
\text { information } \\
\text { query }\end{array}$ & $\begin{array}{c}\text { Unstructured } \\
\text { queries }\end{array}$ & $\begin{array}{c}\text { It is necessary to retrieve the } \\
\text { records that match the key words } \\
\text { by retrieving the keyword and } \\
\text { other methods, and to produce } \\
\text { the corresponding sorting result. } \\
\text { Judge the download behavior } \\
\text { based on the user's different } \\
\text { authorities }\end{array}$ \\
\hline
\end{tabular}

According to the module functional requirements described in Table 1, the system of unstructured data acquisition and retrieval is designed, and the development environment of the system is shown in table 2.

Table 2 Information table of system development environment

\begin{tabular}{cc}
\hline $\begin{array}{c}\text { Environment } \\
\text { name }\end{array}$ & Specific content \\
\hline Hardware & PC, 1.5GB RAM, Intel 2.0GHzCPU \\
environment \\
Software \\
environment \\
$\begin{array}{c}\text { Development } \\
\text { language }\end{array}$ \\
\hline
\end{tabular}

\subsection{Data collection}

In this paper, the unstructured data acquisition document formats are mainly divided into PDF, Excel and so on. In the process of data acquisition, we must ensure that judgment can be made according to the type of upload files, so as to achieve data collection relying on dynamic processing methods ${ }^{[2]}$. For example, in the Word file, you can rely on the resource bank to conduct data storage, and directly obtain the information of the relevant documents according to the corresponding software. However, in the acquisition process, it can be found that POIs are all Java-class bank. Therefore, in order to enable such programs to be successfully applied in data processing, it is necessary to rely on NET CLR-based virtual machine of the open source IKVM.NET. The conversion of data can be completed upon the conversion to NET-class bank. To further demonstrate its practical results, the process of Figure 1 can be used to interpret.

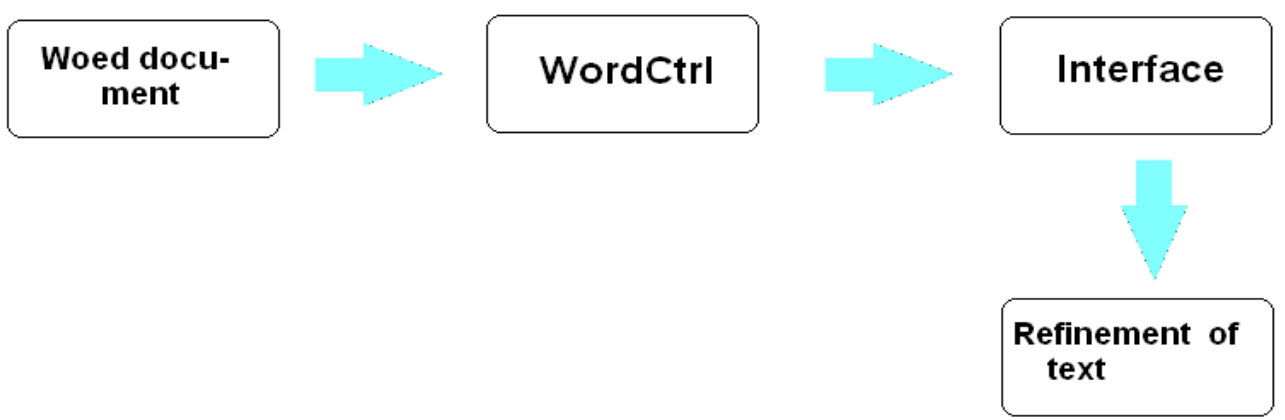

Figure 1 Flow chart of word document information read 


\subsection{The realization of word segmentation algorithm}

For a long time, word bank participle is considered as an ideal Chinese word segmentation method. In the process of unstructured data collection and retrieval, word bank analysis plays an important role in establishing a complete set to match the target information. When it comes across the existing words in the set, the relevant content can be separated. For example, when the word "information security" exists in a word bank, an analyzer can quickly put it into the index as an entry.

According to general data analysis experience, the word segmentation methods to be used is mainly divided into Paoding Segmentation and Pangu Segmentation; and the practice of many scholars also has proved that these two methods can basically meet the basic requirements of the analyzer retrieval. Therefore, according to this result, this article has determined the technical route with the application of the two kinds of word segmentation methods above, which is shown in Fig 2.

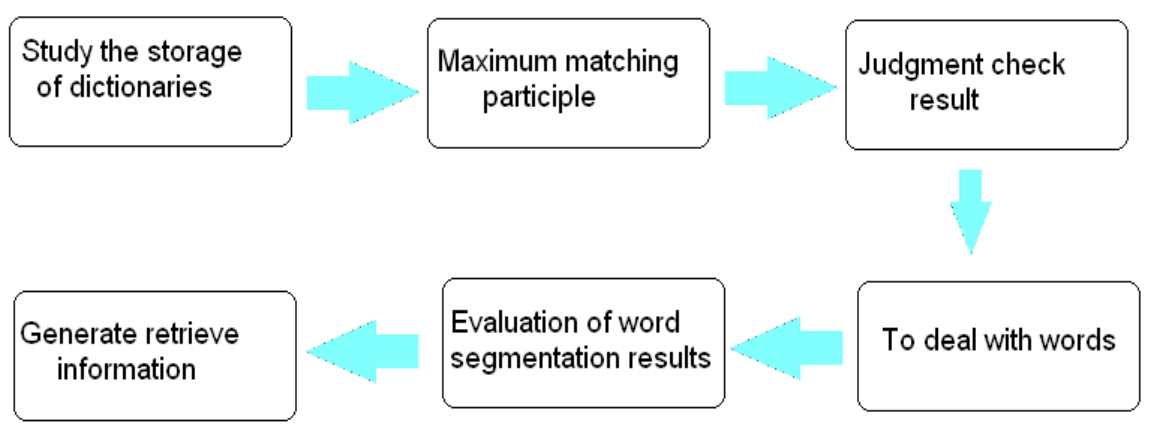

Fig. 2 Road map of algorithm research

According to the algorithm studying route introduced in Fig. 2, we can judge that, in the whole unstructured data retrieval process, the results can be divided into two kinds: the positive matching result and the reverse matching result. In order to deepen the impression of the related personnel, the following hypotheses are presented in this paper.

The contents stored in the target word bank are: high; class; high class; food store; food; store. At this point, input "High class restaurant" to the word bank. The system will firstly match "High-class", and then it goes on to match "food store"; the segmentation process can be interpreted by Table3.

Table 3 The segmentation process of forward maximum matching

\begin{tabular}{cc}
\hline Matched results & String to be matched \\
\hline NULL & high class food store \\
high class & food store \\
high class /food store & NULL \\
\hline
\end{tabular}

Unlike the forward matching, the generation process of reverse matching is different. After inputting "high class food store" in word bank, the system will match the "food store" first, then it goes on to" high class". As shown in table 4.

Table 4 Segmentation process for reverse maximum matching

\begin{tabular}{cc}
\hline Matched results & String to be matched \\
\hline NULL & high class food store \\
food store & high class \\
high class / food store & NULL \\
\hline
\end{tabular}

\subsection{Implementation of full-text retrieval of unstructured data}

Based on the process described above, determination needs to be made from the aspects as follows, so as to achieve full-text retrieval. 


\subsubsection{General condition query}

Taking general document query as an example, after entering the "query" page, the system will load the relevant records of the page by default, and its related information will be displayed in the paging. Each paging will display different data, which is generally divided into three items of data.

In this process, the query conditions are set by the Publisher, the scope of the publication, the title of the file, the effective time, the file form, and so on a variety of content (Figure 3). In this process, the system determines whether the operation of the operator is a "valid operation"

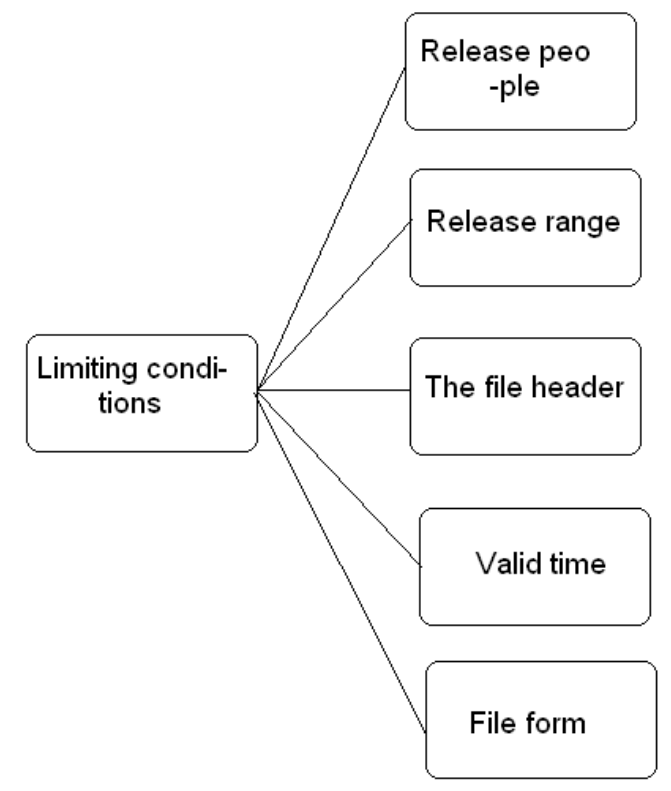

Fig. 3 Source map of general query condition

According to the relevant content of Fig3, after searching the word "government official document", one item that can meet the condition is obtained. At this time the file title can display the matching keyword by special color; and the related personnel can obtain the file relying on the downloading function provided by the system.

\subsubsection{Unstructured query}

In unstructured queries, depending on the "Advanced search" function provided by the system, you also jump to the advanced searching page, then select the label of "Official document" and then type "government documents" into the text box. At this time, the system will use the similarity ranking method to retrieve the information in the thesaurus, and give priority to provide the most matching information, and provide downloads and other services.

In order to improve the quality of search, the improvement can be carried out from the aspects of user query word and analysis of document content. For example, in the retrieval for "microcomputer", "computer" and other words, the system cannot effectively analyze the concept of these two words. So in the process of word identification, a variety of problems often appear. So in unstructured query, special provisions may be provided, so that the relevant people can pay attention to the retrial characteristics of these special terms.

\section{Conclusion}

The unstructured data acquisition and retrieval technology is of great significance to the processing of related technologies. From the perspective of system, this paper studies the acquisition and retrieval techniques of unstructured datasets. In view of the research content, this paper introduces the functions of the modules based on unstructured data acquisition and retrieval system, and graphically shows the contents and data processing points of the system, which is feasible in terms of technology, so it should be attached great importance by relevant personnel. 


\section{References}

[1] Zhuang Z, Qiu T, Zeng X, et al. Research and Application of Acquisition and Processing Technology of Marine Dual Seismic Source Random Shooting[J]. Ship Building of China, 2017, 58:119-126.

[2] Lu Y, Shahabi C, Kim S H. Efficient indexing and retrieval of large-scale geo-tagged video databases [J]. Geoinformatica, 2016, 20(4):1-29. 